INPLASY

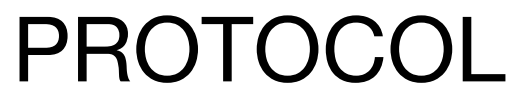

To cite: Chenxi et al. Risk factors of venous thromboembolism in lung cancer patients: A metaanalysis. Inplasy protocol 202080066. doi:

10.37766/inplasy2020.8.0066

Received: 16 August 2020

Published: 16 August 2020

Corresponding author:

Zhao Chenxi

915827717@qq.com

Author Affiliation:

Beijing Chaoyang Hospital, Capital Medical University, Beijing, China

Support: None of financial support

Review Stage at time of this submission: Preliminary searches.

Conflicts of interest:

There is no conflicts of interest.

\section{Risk factors of venous thromboembolism in lung cancer patients: A meta-analysis}

\author{
Zhao, C1; Yao, J2; Ge, Y33.
}

Review question / Objective: What are the risk factors of venous thromboembolism in patients with lung cancer?

Condition being studied: Venous thromboembolism is correlated with increased mortality among lung cancer patients. The characteristics of them presenting with VTE have not been fully established, and our meta-analysis aims to comprehensively investigate the risk factors associated with VTE in patients with lung cancer to help physicians identify VTE earlier in these patients.

Information sources: Multiple databases were searched, including PubMed, EMBASE, Cochrane Library, Web of Science ,China National Knowledge Infrastructure ,VIP and Wanfang.

INPLASY registration number: This protocol was registered with the International Platform of Registered Systematic Review and Meta-Analysis Protocols (INPLASY) on 16 August 2020 and was last updated on 16 August 2020 (registration number INPLASY202080066).

\section{INTRODUCTION}

Review question / Objective: What are the risk factors of venous thromboembolism in patients with lung cancer?
Condition being studied: Venous thromboembolism is correlated with increased mortality among lung cancer patients . The characteristics of them presenting with VTE have not been fully established, and our meta-analysis aims to 
comprehensively investigate the risk factors associated with VTE in patients with lung cancer to help physicians identify VTE earlier in these patients.

\section{METHODS}

Participant or population: (1) studies with a cohort design or case-control design; (2) studies comparing risk factors, including COPD history, pathological type, TNM stage, CVC history, chemotherapy history, WBC, and $\mathrm{Hb}, \mathrm{DD}$, platelet (PLT), $\mathrm{PaO2}$, and CEA levels, and so on; (3) studies involving clear and standardized diagnostic criteria for LC and VTE, including histologically or cytologically confirmed LC, or imaging findings, including echocardiography, computed tomography (CT), magnetic resonance imaging (MRI) or ventilation/ perfusion (V/Q) scan, confirming VTE.

Intervention: Lung cancer patients with Venous thromboembolism (VTE).

Comparator: Lung cancer patients without Venous thromboembolism (VTE).

Eligibility criteria: The quality of the included studies was assessed with the Newcastle-Ottawa Scale (NOS), and a total score of at least 6 was considered high quality. Two reviewers independently evaluated the quality of the eligible conventional studies, and any disagreements were resolved by consensus or discussion with a third author.

Information sources: Multiple databases were searched, including PubMed, EMBASE, Cochrane Library, Web of Science ,China National Knowledge Infrastructure ,VIP and Wanfang.

Main outcome(s): Odds ratios (ORs) and weighted mean differences (WMDs) with $95 \%$ confidence intervals $(95 \% \mathrm{Cls})$ were used as effect measures for dichotomous and continuous variables, respectively.

Quality assessment / Risk of bias analysis: For the quality assessment, the Newcastle-Ottawa Scale(NOS)was used to evaluate all studies, and a total score of at least 6 was considered high quality.

Strategy of data synthesis: Results will be assessed using forest plots and presented as OR for the main outcome and secondary outcomes; An influence analysis will be performed to ascertain the results of the meta-analysis by excluding each of the individual studies. Publication bias will be assessed by a funnel plot for metaanalysis;Statistical analysis will be conducted using Review Manager 5.3 for Mac.

Subgroup analysis: We will consider subgroups such as chemotherapy drugs, surgical operation and PICC.

Sensibility analysis: Exclude articles one by one.

Country(ies) involved: China.

Keywords: lung neoplasms; venous thromboembolism; risk.

Contributions of each author:

Author 1 - Zhao Chenxi - Author 1 contributed to search databases, screen articles and draft the manuscript.

Author 2 - Yao Jiannan - Author 2 contributed to search related studies and analysis statistics.

Author 3 - Ge Yang - Author 3 contributed to the risk of bias and resolve disagreement. 